\title{
Density of Fe-Ni-C liquids at high pressures and implications to liquid cores of Earth and Moon
}

Feng ZHU ${ }^{1}$, XiAOJING LAI ${ }^{1,2}$, JiAnWEI WANG ${ }^{3}$, GEORGE AMULELE $^{1}$, YOSHIO KONO ${ }^{4}$, BIN CHEN ${ }^{1}$

${ }^{1}$ Hawai' $i$ Institute of Geophysics and Planetology, University of Hawai'i at Mānoa, Honolulu, Hawai'i 96822, USA

${ }^{2}$ Gemmological Institute, China University of Geosciences, Wuhan, Hubei, China.

${ }^{3}$ Department of Geology and Geophysics, Louisiana State University, Baton Rouge, LA 70803, USA

${ }^{4}$ HPCAT, Advanced Photon Source, Argonne National Laboratory, Argonne, IL 60439, USA

The presence of light elements in the metallic cores of Earth, Moon and other terrestrial bodies has been widely proposed. Carbon is among the top candidates in light of its high cosmical abundance, siderophile nature and ubiquity in iron meteorite. It is however still under debate whether carbon as the major light element can account for the seismic observations in Earth's or lunar cores, mainly due to the controvorsy whether incorporation of carbon can increase or decrease the compressional sound velocity $\left(v_{p}\right)$ of liquid FeNi alloy between density and sound velocity measurement. (Terasaki et al. 2010; Shimoyama et al. 2013, 2016; Nakajima et al. 2015). Here we report density and elasticity of liquid Fe90Ni10-3 wt.\% $\mathrm{C}$ and $\mathrm{Fe}_{90} \mathrm{Ni}_{10}-5 \mathrm{wt}$ \% $\mathrm{C}$ alloys using the synchrotron-based X-ray absorption experiments and density functional theory (DFT) calculations. The results show that the alloying of 3-5 wt.\% $\mathrm{C}$ lowers the density of $\mathrm{Fe}_{90} \mathrm{Ni}_{10}$ liquid by $\sim 2.9-3.1 \%$ at $\sim 2 \mathrm{GPa}$, and $\sim 3.4-3.6 \%$ at $\sim 9 \mathrm{GPa}$. More intriguingly, the bulk moduli of the $\mathrm{Fe}-\mathrm{Ni}-\mathrm{C}$ liquids was found to be similar to or slightly higher than Fe-Ni alloy in both experiments and calculations. Thus, $v_{p}$ of Fe-Ni-C alloy is calculated to be higher than pure $\mathrm{Fe}-\mathrm{Ni}$ alloy, favoring carbon as a strong candidate to explain the elevated $v_{p}$ in Earth's outer core. On the other hand, the high $v_{p}$ of Fe-Ni-C liquids does not match the reported $v_{p}$ of lunar liquid core, suggesting that carbon may not be the dominant light element in Moon's liquid core.

\section{References}

Terasaki et al. (2010) Journal of Geophysical Research: Solid Earth, 115(B6).

Shimoyama et al. (2013) Physics of the Earth and Planetary Interiors, 224, 77-82.

Nakajima et al. (2015) Nature communications, 6, 8942.

Shimoyama et al. (2016) Journal of Geophysical Research: Solid Earth, 121(11), 7984-7995. 